\title{
CkREV enhances the drought resistance of Caragana korshinskii through regulating the expression of auxin synthetase gene CkYUC5
}

Chunmei Gong ( $\nabla$ gcm228@nwafu.edu.cn )

Northwest Agriculture and Forestry University https://orcid.org/0000-0002-1164-5440

Jiayang Li

Northwest A\&F University: Northwest Agriculture and Forestry University

Jiejie Ren

Northwest A\&F University: Northwest Agriculture and Forestry University

Tianxin Zhang

Northwest A\&F University: Northwest Agriculture and Forestry University

Jinhao Cui

Northwest A\&F University: Northwest Agriculture and Forestry University

\section{Research Article}

Keywords: Drought,Auxin synthesis,HD-ZIP III,Bidirectional regulation,Stress resistance

Posted Date: February 11th, 2022

DOI: https://doi.org/10.21203/rs.3.rs-1288023/v1

License: (c) (1) This work is licensed under a Creative Commons Attribution 4.0 International License. Read Full License 


\section{Abstract}

As a common abiotic stress, drought severely impairs the growth, development and even sur-vival of plants. Here we report a transcription factor, Caragana korshinskii REVOLUTA(CkREV), which can bidirectionally regulate the expression of the critical enzyme gene $C k Y U C 5$ in auxin synthesis according to external environment changes, so as to control the biosynthesis of auxin and further enhance drought resistance of plants. Quantitative analysis reveals that the expression level of $C k Y U C 5$ and AtYUC5 is both down-regulated after $C$. korshinskii and Arabidopsis thaliana are exposed to drought. Functional verification of CkREV reveals that CkREV up-regulates the expression of AtYUC5 in transgenic A. thaliana under common conditions, while down-regulates it under drought conditions. Meanwhile, the expression of $C k Y U C 5$ is also down-regulated in $C$. korshinskii leaves instantaneously overexpressing CkREV. We apply dual-luciferase reporter system to discover that CkREV can bind to the promoter of CkYUC5 to regulate its expression, which is further proved by EMSA and $\mathrm{Y} 1 \mathrm{H}$ experiments. Functional verification of CkREV in $C$. korshinskii and transgenic $A$. thaliana shows that CkREV can regulate the expression of CKYUC5 and AtYUC5 in a contrary way, maintaining the equilibrium of plants between growth and drought resisting. CkREV can positively regulate the expression of $C k Y U C 5$ to promote auxin synthesis in favor of growth under normal development. However, CkREV can also respond to external signals and negatively regulate the expression of $C k Y U C 5$, which inhibits auxin synthesis in order to reduce growth rate and lower water demands and eventually improves drought resistance of plants.

\section{Key Message}

CKREV depends on the change of external environment to regulate the expression of CkYUC5 to balance plant growth and stress resistance.

\section{Introduction}

Auxin primarily originates from Greek meaning growth (Salehin et al. 2015). It is irreplaceable during plant growth and development, which influences plant apical growth, axillary bud formation, floral organ development and root development (Brumos et al. 2018; Bu et al. 2020; Olatunji et al. 2017; Zhao 2010). On the cell level, auxin is competent changing the plasticity of plant cells, facilitating cells to differentiate and elongate. Besides, auxin can also compose a sophisticated regulatory network together with diverse kinds of plant hormones, collectively accommodate plants to their surroundings (Vanstraelen and Benková 2012). Auxin biosynthesis mainly depends on tryptophan pathway consisting of four chief branches, each of which can synthesize indoleacetic acid (IAA) catalyzed by different enzymes (Casanova-Sáez and Voß 2019; Olatunji et al. 2017; Zhao 2010). So far, only IpyA pathway has been clearly proved. It mainly includes two steps. First, tryptophan is reversibly transformed to IpyA through transamination catalyzed by TRYPTOPHAN AMINOTRANSFERASE OF ARABIDOPSIS (TAA). Then, IpyA is irreversibly transformed to IAA through oxidative decarboxylation by YUCCA (YUC), a rate-limiting enzyme (Zhao et al. 2001; Zheng et al. 2013). TAA/YUC pathway has been functionally proved in various plants serving as the chief biosynthesis pathway for endogenous auxin (Pacheco-Villalobos et al. 2013; Phillips 
et al. 2011; Stepanova et al. 2011; Yoshikawa et al. 2014; Zhao 2018). YUC5, one protein from YUC family, plays an important role in regulating auxin biosynthesis.

ABA, salicylic acid and ethylene are involved in plant's responses to drought stress, so do auxin (Chen et al. 2021; Rowe et al. 2016). Under stress conditions, WES1, a gene encoding IAA-amido synthase, from GH3 gene family is up-regulated to inactivate IAA by binding it to an amino acid, which lowers the level of endogenous auxin and activates the expression of stress-related genes $P R-1$ and $C B F$ (Park et al. 2007). Aux/IAA and ARF are two significant protein families mediating auxin response, which directly regulate the expression of auxin early response genes. 31 OsIAA genes and 25 OSARF genes have been identified in Oryza sativa, among which OSIAA2 and OsIAA20 are up-regulated under high salinity conditions. The expression level of 0 . sativa TLD1 from GH3.13 gene family is inhibited in tissues above ground under normal conditions while is remarkably induced under drought conditions. The activation of TLD1 gene leads to the reduce in IAA concentration and the change of plant shape in 0 . sativa t/d1-D gain-of-function mutant, which decreases plant water loss and improves survival rate (Zhang et al. 2009). During seed germination, $n t m 2-1$ mutant possesses strong salt resistance. Salt stress can induce NTM2 to specifically bind to the promoter of IAA3O and activate its high expression. Nevertheless, the high expression of IAA3O induced by $\mathrm{NaCl}$ disappears in ntm2-1 mutant, which attenuates the inhibition of auxin on seed germination (Park et al. 2011). Moreover, TCPs are able to activate the expression of auxin synthesis genes, for example YUC8, by facilitating the transcription activity of PIF4 under high temperature stress (Han et al. 2019).

HD-ZIP III family significantly regulate the pattern formation of embryo, root, stem and vascular bundle together with leaf development (Bowman and Floyd 2008; Carlsbecker et al. 2010; Smith and Long 2010). It was first reported in 1995 that $A T H B$ 8, a member of HD-ZIP III family in $A$. thaliana, could express after induced by auxin (Baima et al. 1995). Further researches uncovered that auxin flow induced the expression of MONOPTEROS (MP), the latter would induce the expression of PIN-FORMED 1 (PIN1), and polarly localized PIN1 in high expression level would promote the polarity flow of auxin. MP directly binds to the promoter of $A T H B 8$ to induce its expression while inducing PIN1. Interestingly, up-regulated AtHB8 reduces the sensitivity of MP-induced PIN1 to auxin, thus limits auxin flow to a narrow scope (Donner et al. 2009)and stimulates precursor cells of procambium to differentiate into xylem cells at designated regions. The expression of other HD-ZIP III members, such as ATHB15, PHV, PHB and REV/IFL, are downregulated in $m p$ mutants, which can be considered to be regulated by MP (Mattsson et al. 2003). Besides, auxin biosynthesis genes TAR1, TAR2 and YUCCA3, 5, 7, 8, 9 are indispensable for high expression of HDZIP III in primary root and the formation of metaxylem in A. thaliana (Ursache et al. 2014). Meanwhile, HD-ZIP III family shares the same expression pattern with auxin (Floyd and Bowman 2006; Floyd et al. 2006; Heisler et al. 2005). Hence, the achievement of the function of HD-ZIP family possesses close relationship with auxin.

Researches focusing on the relationship between auxin and plant response to stress have been increasingly emphasized in recent years. Studies concentrating on the relationship between drought response genes and plant hormones in $A$. thaliana unveil that although ABA-dependent pathway 
dominates plant response to drought stress, other plant hormones including auxin also have impact on the expression of genes related to drought resistance (Huang et al. 2008). Abundant studies have indicated that the synthesis and critical response genes of auxin are regulated by environmental stress, however, the response to stress of auxin and the regulatory mechanism thereof at molecular level still require to be elucidated. This paper reports a transcription factor called CkREV, a member of HD-ZIP III family which can bidirectionally regulate the expression of the critical enzyme CkYUC5 in auxin synthesis according to external environment changes in C. korshinskii, a drought resisting pioneer plant widely spread among the desert area in northwest China, and its mechanism of maintaining the equilibrium of plants between growth and drought resisting by controlling auxin biosynthesis.

\section{Materials And Methods Plant Materials}

C. korshinskii samples employed originated from the experimental plot of Northwest A\&F University, which were cultivated in soil after germination. Healthy and plump seeds were selected and washed by clean water. Then seeds were wrapped up with wet gauze and left in dark environment for germination for 3-5 days under room temperature before being transplanted into flowerpots.

A. thaliana samples employed were all Col-0 ecotype. The $p 35$ S.CkREV-GFP and $p 35$ S.GFP plasmids were transformed into Agrobacterium tumefaciens GV3101, which was used to infect $A$. thaliana of Col-0 ecotype. Seeds were disinfected by $10 \%(\mathrm{v} / \mathrm{v})$ sodium hypochlorite for 5 minutes and were sifted on $1 / 2$ MS medium with hygromycin until homozygous $\mathrm{T}_{3}$ generation.

\section{qPCR Measurement}

The total RNA of plant tissue was extracted by Plant RNA Isolation Kit (Beibei Bio, China). For each sample, we accurately absorbed $1 \mu \mathrm{g}$ RNA according to RNA concentration of different treatments and repetitions. The first strand of cDNA was amplified by PrimeScriptTM RT reagent Kit with gDNA Eraser (Takara, Japan), and cDNA solution was diluted for 3-5 times as the template of qRT-PCR. Quantitative analysis on the expression of relevant genes was performed on $2 \times$ M5 HiPer Realtime PCR Super mix (Mei5bio, China) and quantitative PCR Amplifier LightCycler 480 (Roche, Switzerland). Quantitative primers are listed in table S2.

\section{Genome walking}

We designed 3 specific $R$ primers ( SP1: TACCTAGCAGACTGAACACACTCGT, SP2:

GAAGATGAAGCTTTAATCGGTCGTA and SP3: TCTCTTAGGCGTACTGCTGTGGCTA), among which SP2 should be designed within SP1 and SP3 within SP2. There was no stringent requirements on the distance between every two primers, as 60-100 bp is preferable normally. Genome walking usually consists of 
nested PCR reaction for three rounds, each of which is thermal asymmetric interlaced PCR in two different annealing temperature. Higher annealing temperature encourages the binding of specific primers while lower one benefits that of universal primers. The sequence of universal primer and amplification programme employed in genome walking comply with that in literature cited (Liu and Chen 2007).

\section{GUS staining}

The CkYUC5 promoter was amplified from C. korshinskii genomic DNA and inserted into pCambia 1305 vector. $p 35 s$ ::CkREV-GFP and p35S.:GFP as effector plasmids were co-injected with pCkYUC5::GUS into tobacco leaves and cultured for 48 hours. After different treatments, the GUS staining kit (Coolaber, China) was used to stain and observe the GUS expression level in the tip of the tobacco leaf.

\section{IAA content detection}

Four days after germination on $1 / 2 \mathrm{MS}$ plates, the $A$. thaliana seedlings under normal culture conditions and PEG treatment for 3 days. Accurately weighing $0.1 \mathrm{~g}$, after fully ground with liquid nitrogen, $0.9 \mathrm{~mL}$ of PBS ( $\mathrm{pH} \mathrm{7.4)} \mathrm{solution} \mathrm{was} \mathrm{added} \mathrm{to} \mathrm{dissolve} \mathrm{at} \mathrm{a} \mathrm{ratio} \mathrm{of} \mathrm{1:9} \mathrm{(w/v).} \mathrm{After} 20 \mathrm{~min}$ at room temperature, centrifuged at $3000 \mathrm{rpm}$ for $20 \mathrm{~min}$, and the IAA content was detected by Plant Indole-3-acetic acid(IAA); Auxin ELISA Kit. (Jingmei, China).

\section{Dual-LUC assay}

The promoters of target genes were amplified from the genome DNA of $C$. korshinskii and inserted into p Green II 0800-LUC vector with $p 35 s$ ::CkREV-GFP and $p 35 s:$ :GFP used as effect plasmids. Reporter and effector were transformed into Agrobacterium tumefaciens GV3101 (pSoup-p19) and GV3101 respectively, which were cultured in shaking incubator under constant temperature until OD value was around 1.0. Bacteria were resuspended in solution with acetosyringone (AS) to OD 600 value was 0.8 . Effector and reporter were combined in the proportion 8:2 and placed in dark environment for activation for 2-4 $\mathrm{h}$ before injected into leaves of Nicotiana tabacum at the age of $28 \mathrm{~d}$. Samples were collected to test the activity of firefly luciferase and renilla luciferase by GloMax 20/20 Luminometer (Promega, USA) and Dual luciferase reporter assay system (Promega, USA) reagents according to their instructions (Tu et al. 2020).

\section{Electrophoretic mobility shift assay(EMSA)}

First, biotin label was linked to the $3^{\prime}$ end of artificially synthesized single-stranded oligonucleotide probe containing binding site by DNA 3' end biotin label kit (Beyotime, China). Second, double-stranded DNA 
probe with biotin label was obtained by annealing with artificially synthesized complimentary chain. Third, purified CkREV protein was incubated with probe with biotin label at a certain proportion while unlabeled double-stranded probe was used as cold probe. Fourth, native-PAGE was employed to separate samples before transferred onto nylon membrane (Solarbio, China) with positive charge through wet transformation method. Fifth, the nylon membrane was placed under ultraviolet cross linker purple (UVP, USA) at $254 \mathrm{~nm}, 120 \mathrm{~mJ} / \mathrm{cm}^{2}$ for $60 \mathrm{~s}$. Last, colour development was employed on completely crosslinked nylon membrane by EMSA chemiluminescence kit (Beyotime, China) for observation under chemiluminescence imager. Detailed steps complied with instructions of EMSA chemiluminescence kit from Beyotime Company.

\section{Yeast one-hybrid}

The transcription factor was connected to the $p G A D T 7-r e c 2$ vector, and the promoter fragment to be verified was connected to the $p H I S 2$ vector. The above plasmids were co-transformed into yeast strain Y187 using the lithium acetate method, and screened by SD/-LT medium. The positive colonies that were successfully transformed were picked out in YPDA liquid medium, cultivated at $30^{\circ} \mathrm{C}$ to $O D 1.0$ and diluted to 1:100 and 1:1000 three concentration gradients. We spotted the bacteria liquid on SD/-LTH plates containing 10-100 mM 3-AT for self-activation verification, and performed experiments on the plate with 3-AT, the concentration of which inhibits self-activation.

\section{DAB and NBT staining}

DAB can be oxidized by hydrogen peroxide into dark brown precipitates. Hence, DAB is employed as dye to test the existence and distribution of hydrogen peroxide in plant cells. DAB solution was prepared at the concentration of $1 \mathrm{mg} / \mathrm{mL}$ and acidated by $0.2 \mathrm{M} \mathrm{HCl}$ to pH 3.0. $5 \mu \mathrm{L}$ TWEEN-20 $(0.05 \% \mathrm{v} / \mathrm{v})$ and 0.5 $\mathrm{mL} 200 \mathrm{mM} \mathrm{Na}_{2} \mathrm{HPO}_{4}$ were added into DAB solution while stirring, which produced DAB staining solution of $10 \mathrm{mM} \mathrm{Na}{ }_{2} \mathrm{HPO}_{4}$ and increased $\mathrm{pH}$ again. Leaves were collected and absolutely immersed into DAB staining solution under vacuum and dark conditions for shake incubation for 4-5 $\mathrm{h}$ at the speed of 80-100 rpm, after which DAB solution was discarded and samples were bleached by solution (ethanol:acetic acid:glycerol=3:1:1) before being photographed (Daudi and O'Brien 2012).

For NBT staining, leaves were immersed into $6 \mathrm{mM}$ NBT solution prepared by citrate sodium buffer $(\mathrm{pH}$ 6.0) under vacuum and dark conditions for incubation for 5-8 h, after which NBT solution was discarded and samples were bleached by solution (ethanol:acetic acid:glycerol=3:1:1) before being photographed (Kaur et al. 2016).

\section{Results}

\section{HD-ZIP III TFs phylogenetic analysis}


We constructed a phylogenetic tree including transcription factors of HD-ZIP III family in C. korshinskii, Glycine max, Cicer arietinum, Medicago truncatula, Cajanus cajan, Camellia sinensis and $A$. thaliana (Fig. 1). 31 HD-ZIP III proteins derived from different species were categorized into four branches, among which the members of HD-ZIP III family in C. korshinskii exhibited relatively close relationship with those in G. max and M. truncatula, located in the same branch. On the contrary, REV exhibited relatively distant relationship with other members of HD-ZIP III family, implying it probably possessed unknown regulatory functions different from others. We analyzed the conserved domain of the transcription factors of the HDZIP III family members of the above species through the pfam (http://pfam.xfam.org/). The HD-ZIP III family of $C$. korshinskii including CkREV, has basically the same number and distribution of motif as other species (Fig. 2), and CkREV subcellular localization is also in the nucleus (Fig. 3), which indicates that they may have similar biological functions.

\section{CkREV balances plant growth and stress resisting by regulating the expression of CkYUC5}

Under stress conditions, WES1, a gene encoding IAA-amido synthase, from GH3 family is up-regulated to inactivate IAA by binding it to an amino acid, which lowers the level of endogenous auxin (Chen et al. 2021). In addition to scavenging the existing IAA, can plants enhance their stress resistance by reducing its biosynthesis under stress? First, we treated $C$. korshinskii with drought conditions in order to explore the expression of the critical enzyme gene CkYUC5 in auxin synthesis. We performed RT-qPCR to detect the expression level of CkYUC5 (Fig. 4a), which suggested that CkYUC5 was down-regulated under drought conditions and auxin synthesis may be inhibited. The same outcomes were proved in $A$. thaliana, it dropped precipitously with increasingly severe drought treatment (Fig. 4b). In A. thaliana, the HD-ZIP III family has the same expression pattern as auxin (Floyd and Bowman 2006; Floyd et al. 2006; Ursache et al. 2014). Auxin biosynthesis genes TAR1, TAR2 and YUCCA3, 5, 7, 8 and 9 are necessary for the high expression of HD-ZIP III family and the formation of metaxylem in $A$. thaliana primary roots (Mattsson et al. 2003), Nevertheless, a member of the HD-ZIP III family called REV can directly bind to the promoter region of YUC5 (Brandt et al. 2012) and $\angle A X 2,3$ to regulate the synthesis and transport of auxin respectively (Baima et al. 2014). These two processes are closely related to the HD-ZIP III family, but the crosstalk between them under stress conditions has not been reported. In this study, we discovered that the expression level of CkREV, a member of HD-ZIP family, was continuously up-regulated with increased drought level (Fig. 4c). Whether CkREV mediates the negative regulation of CkYUC5 or not?

We overexpressed CkREV in A. thaliana and performed qRT-PCR to test the expression level of AtYUC5 in transgenic $A$. thaliana, the outcome of which indicated that the expression level of AtYUC5 in CkREV-OE lines was conspicuously up-regulated (Fig. 4d). Meanwhile, we instantaneously transformed CkREV into C. korshinskii leaves through plasmolysis and deplasmolysis to test the expression level of endogenous auxin synthetase gene CkYUC5 after CkREV was overexpressed. Intriguingly, the expression level of CkYUC5 is considerably down-regulated by CkREV (Fig. 4e). The method we used to transform CkREV into C. korshinskii leaves caused osmotic stress similar to drought stress, which brought about a question 
whether the contrary regulation mechanisms in CkREV stably transformed $A$. thaliana and instantaneously transformed original plant were the consequences of osmotic stress during transformation process. We made further efforts to raise wild type and CkREV-transformed $A$. thaliana under drought conditions and perceived that the expression of AtYUC5 was remarkably down-regulated by CkREV under drought response (Fig. 4f) while it was exceedingly up-regulated in normally cultured $A$. thaliana CkREV-OE lines.

\section{CkREV affects auxin biosynthesis by regulating CkYUC5 and inhibiting $A$. thaliana root length under stress.}

The promoter of $C k Y U C 5$ was constructed to the pCambia1305 vector, and the expression pattern of CkYUC5 gene was observed by the expression of $\beta$-glucuronidase (GUS) gene. Under normal culture condition $C k Y U C 5$ was abundantly expressed in the tip of tobaco leaves, which could be further upregulated by the overexpression of CkREV. Consistent with the previous RT-qPCR results, the accumulation of GUS signals guided by the CKYUC5 promoter was significantly inhibited after PEG treatment (Fig. 5a-d and h). The root length of transgenic $A$. thaliana after PEG treatment was further analyzed. Under normal culture condition, the $A$. thaliana CKREV-OE line showed no significant difference in root length compared with the wild-type, but after PEG treatment, the root length of $A$. thaliana CkREV$\mathrm{OE}$ line was inhibited (Fig. $5 \mathrm{e}$ and f). In A. thaliana, the balance between cell division and differentiation depends on the mutual regulation of hormone cytokinin and auxin (Dello loio et al. 2012). A previous study found that the free IAA content in the roots of $A$. thaliana yucQ mutants decreased by $55 \%$ compared to the wild type, and the lack of auxin significantly inhibited root elongation (Chen et al. 2014). In order to further verify whether the inhibition of root length of the $A$. thaliana CKREV-OE line after PEG treatment was related to auxin deficiency, the transgenic $A$. thaliana was treated with PEG while adding $0.05 \mathrm{mg} / \mathrm{L}$ NAA, and the root length of $A$. thaliana CKREV-OE line returned to the level of wild type (Fig. $5 \mathrm{~g}$ and i). At the same time, the auxin content was detected in the $A$. thaliana seedlings treated as above. CkREV overexpression did not induce the accumulation of IAA under normal culture conditions, but after drought stress treatment, the IAA content in the CKREV-OE line was decreased and significantly lower than WT (Fig. 5j and S3). In summary, CkREV affects the biosynthesis of auxin on the expression of CkYUC5 in different environment, and plays an important role in regulating plant growth and stress adaptation.

\section{CkREV interacts with the promoter of CkYUC5 to regulate its expression}

The following critical question consists in whether CkREV directly or indirectly regulates CkYUC5. Since there was no public genome information about $C$. korshinskii, we firstly cloned the promoter of $C k Y U C 5$ by genome walking and successfully obtained the unknown promoter sequence for $1144 \mathrm{bp}$ in total upstream from CkYUC5 after two rounds of nested PCR (Fig. S1). We predicted transcription factor families probably regulating CkYUC5 promoter region by PlantTFDB (http://planttfdb.gao-lab.org/) and 
found 27 appropriate ones in aggregate, among which members of ERF and WRKY family extensively regulated that region (Table S1), in which a large quantity of light response elements were predicted by PlantCare as well (Fig. S2). However, we also discovered that transcription factors from HD-ZIP family potentially regulated it. We found that ATGAT is necessary for the binding of AtREV by searching its binding site on PLANT PAN (http://plantpan.itps.ncku.edu.tw/) (Fig. 6a). Being homologous gene of AtREV, CkREV possesses relatively conservative binding site. We constructed Dual-luciferase reporter system with cloned CkYUC5 promoter (Fig. 6b), and injected it into Nicotiana tabacum to detect the activity of firefly luciferase and renilla luciferase. It turned out that CkREV could interact with CkYUC5 and the former negatively regulated the expression of the latter remarkably (Fig. 6c). We also discovered that the promoter region of CKYUC5 possessed ATGAT element, namely the binding site of CkREV. Based on that, we synthesized probes containing the core element and adjacent sequence (Fig. 6d) with biotin label linked to its $3^{\prime}$ end and cold probes without biotin label for EMSA experiments in order to prove their interaction (Fig. 6e). It turned out that CkREV could bind to the probes with biotin label while cold probes competed with them. Therefore, it is proved that CkREV can bind to ATGAT element in the promoter region of $C k Y U C 5$ and regulate its expression. At the same time, we also used the yeast one-hybrid method for supplementary verification (Fig. 6f), which further confirmed the regulation of CkREV on CkYUC5.

Interestingly, as a transcriptional activator, CkREV can negatively regulate the expression of CkYUC5. We speculate that there are other transcription factors involved in the regulation of CkREV. CkAS1, which is closely related to the function of CkREV, encodes a R2-R3 MYB domain protein that inhibits transcription. The results of qRT-PCR found that under PEG-simulated drought conditions CkAS1 in C.korshinskii leaves was significantly up-regulated, which is seen as CkREV was transiently overexpressed as well (Fig. $6 \mathrm{~g}$ ). Therefore, CkAS1 may be involved in the negative regulation of CkREV on CkYUC5. On the basis of the previous Dual-LUC experiment, we injected two effectors CkAS1 and CkREV into tobacco and detected the expression of reporter gene. Compared with CkREV alone CkREV+CkAS1 further down-regulated the expression of $C k Y U C 5$ (Fig. 6h and i).

\section{CkREV down-regulates the expression of YUC5 to enhance the drought-resisting ability of plants under drought response}

Based on the results above, we discovered that CKREV would respond to external signals with improved expression level when plants were confronted with drought stress. Its regulation on the expression of CkYUC5 would transfer from positive to negative, which participates in the process of auxin reduction under stress conditions. So, what contributions do negative regulation of CkREV on auxin synthesis make to adaptation of plants to drought stress? We treated transgenic $A$. thaliana with drought and detected the level of ROS in leaves by DAB (Fig. 7a) and NBT (Fig. 7b) staining. It turned out that $A$. thaliana CKREV-OE lines possessed ROS at a considerably lower level under drought stress. We also determined several drought resistance indexes for transgenic $A$. thaliana, such as proline content (Fig. 7c) and 
relative water content (Fig. 7d), the results of which indicated that CkREV-OE lines possessed rather exceptional traits for drought resistance, exhibiting excellent capability to adapt to drought stress. In summary, CkREV is conducive to the biosynthesis of auxin and the acceleration of plant growth by positively regulating the expression of $C k Y U C 5$ gene during normal development. However, when plants are subjected to drought stress, CkREV negatively regulates the expression of $C k Y U C 5$ by sensing external signals and inhibits the biosynthesis of auxin, thereby slowing the growth rate of plants, reducing their demand for water and enhancing the ability of plants to adapt to drought.

\section{Discussion}

The synthesis and metabolism, homeostasis regulation, polar transport and signal transduction of auxin collectively influence its distributing gradient and how plants respond to it, which plays an indispensable role throughout the growth and development of plants. Auxin was primarily discovered to facilitate the growth of stems and roots (Salehin et al. 2015). Gradually, people perceived that it impacted a variety of physiological activities in plants, including senescence controlling, responses to abiotic stress and pathogens, formation of fruits, establishment and maintenance of cell polarity, apical dominance, phototropism and geotropism etc (Brumos et al. 2018; Bu et al. 2020; Leyser 2018; Ramos Báez and Nemhauser 2021; Retzer and Weckwerth 2021; Wang et al. 2010). Auxin is synthesized in immature tissues such as tender leaves, cotyledons and roots (Blakeslee et al. 2019; Ikeda et al. 2009; Stepanova et al. 2008; Yamada et al. 2009; Yang et al. 2014). For roots, the synthesis of auxin aids in maintenance of its concentration gradient, which is tremendously significant for normal growth and development (Olatunji et al. 2017).

Auxin also possesses close relationship with stress. Recent researches have indicated that growth retardation is the direct consequence of osmotic stress (Skirycz and Inzé 2010). Early in the 1970s, studies showed that plants with smaller cells possessed stronger capability of resisting low water potential and water deficiency. Hence, rapid reaction to environmental changes and self-restriction on growth rate probably benefit plants to survive dry period (Naser and Shani 2016). Direct determination on the content of endogenous IAA in leaves and roots indicates that plants living under salt stress and water deficiency conditions possess IAA at a apparently low level (Du et al. 2013; Liu et al. 2015). Further studies reveal that $O$. Sativa possesses seven YUC genes for auxin biosynthesis, six of which exhibit low expression level under dry conditions (Du et al. 2013). Studies focusing on the relationship between drought response genes in $A$. thaliana and plant hormones uncover that near 100 genes can respond to drought stress while responding to IAA (Huang et al. 2008). The expression of TLD1 from GH3.13 gene family in $O$. Sativa is tremendously induced under drought stress. In $O$. Sativa t/d1-D gain-of-function mutant, the activation of TLD1 gene leads to the decrease of IAA concentration and the change of plant shape, which abates water loss and improves survival rate (Zhang et al. 2009).

For members of HD-ZIP family, we discovered that they could bidirectionally regulate auxin synthesis under normal and drought conditions (Fig. 8). HD-ZIP III family shares the same expression pattern with auxin (Floyd and Bowman 2006; Floyd et al. 2006; Heisler et al. 2005), the function of which is closely 
related to the synthesis and transport of auxin. The expression level of CKREV from HD-ZIP III family in drought-resistant shrub $C$. korshinskii is continuously up-regulated in response to drought stress while that of $C k Y U C 5$ is significantly down-regulated (Fig. 4a and c). We further performed functional verification on $C K R E V$ by stably transforming it into $A$. thaliana and instantaneously transforming it into C. korshinskii, which revealed that the expression level of AtYUC5 was tremendously up-regulated in $A$. thaliana CKREV-OE lines while that of CKYUC5 was significantly down-regulated in $C$. korshinskii after instantaneous transformation (Fig. $4 \mathrm{~d}$ and e). We inferred that instantaneous transformation of $C k R E V$ into $C$. korshinskii leaves by plasmolysis and deplasmolysis imposed osmotic stress similar to drought stress upon them, thus CKREV regulated the expression of $C k Y U C 5$ and AtYUC5 in contrary mechanisms. Later, we proved the inference above by treating transgenic $A$. thaliana with drought, which showed that CKREV significantly down-regulated the expression of AtYUC5 (Fig. 4f).

The results of GUS staining in tobacco leaves also proved the above results, and the regulation of CkYUC5 by CkREV was closely related to drought stress. CkREV participates in the negative regulation of auxin biosynthesis by down-regulating the expression of $C k Y U C 5$ under drought stress, which reduces the growth rate of plants under drought stress (Fig. 5). Further experiments such as Dual-LUC, EMSA and Y1H all confirmed that $C K R E V$ could interact with the promoter of $C k Y U C 5$ by binding to ATGAT element to directly regulate its expression (Fig. 6). Interestingly, as a transcriptional activator, CkREV can negatively regulate the expression of $C k Y U C 5$, which is proved by qRT-PCR and Dual luciferase reporter system that CkREV promotes the accumulation of CkAS1 in C. korshinskii leaves prior to its participation in the negative regulation process of CkREV on CkYUC5 (Fig. 6g-i).

The expression level of auxin is stringently regulated in plants. Abundant studies reveal that asymmetrical distribution of auxin is critical for plant development (Paque and Weijers 2016). Overexpression of ZmPIN1a in Zea mays lowers the height of maize, increases the number of lateral roots and inhibits their elongation, which assists to form a well-developed root system and which improves its resistance to drought, lodging and low phosphate environment (Li et al. 2018). Under stress conditions, the expression of WES1 gene encoding IAA-amido synthase from $\mathrm{GH} 3$ family is up-regulated, deactivating IAA by catalyzing its binding to an amino acid, which enhances plant resistance through activating the expression of stress-related genes $P R-1$ and $C B F$ by lowering the level of endogenous auxin (Park et al. 2007). We utilized DAB and NBT staining to detect the level of ROS in $A$. thaliana leaves after drought treatment while determining relevant physiological indexes, the results of which indicated that $A$. thaliana CKREV-OE lines suffered less under drought conditions, exhibiting capability for drought adaptation (Fig. 7).

In conclusion, the distinct expression patterns of YUC5 under diverse environment explained that auxin biosynthesis in plant was stringently regulated. CkREV responded to external environment changes and further influenced the expression of $C K Y U C 5$ and AtYUC5 in contrary ways, indicating that extremely the sensitivity of CkREV to the environment determines regulatory directions of its downstream genes. Accordingly, CkREV can enhance the expression of $C k Y U C 5$ in favor of plant growth during normal development while it can sense external signals to function conversely in order to decelerate plant growth 
and attenuate water demands confronted with drought stress. This research provides a novel pathway for expanding the nature of drought-resisting in $C$. korshinskii, offering choices of functional and regulatory genes for enhancing the drought-resistance of woody plants through biotechnology in desert area from now on.

\section{Declarations}

\section{Acknowledgments}

We thank the National Natural Science Foundation of China for funding this work (No.31770648 and 31070538); We thank the Key R\&D Projects in Shaanxi Province for funding this work (2020NY-190); We earnestly thank everyone in Tea Plant Development and Stress Biology Laboratory of Northwest A\&F University for their assistance. We are grateful to Miss Beibei He, Miss Minrong Luo and Mr Fei Zhang (Horticulture Science Research Center, Northwest A\&F University, Yangling, China) for their assistance with microscopy and other analysis.

\section{Author Contributions}

Experiments in this study were designed by GCM and LJY. LJY accomplished the majority of experiments and the writing of the manuscript. RJJ fostered plant materials and provided several experimental ideas. ZTX provided several experimental ideas. CJH translated entire paper. All authors have read and agreed to the published version of the manuscript.

\section{Conflicts of Interest}

The authors declare no conflict of interest.

\section{References}

1. Baima S, Forte V, Possenti M, Peñalosa A, Leoni G, Salvi S, Felici B, Ruberti I, Morelli G (2014) Negative feedback regulation of auxin signaling by ATHB8/ ACL5-BUD2 transcription module. Mol Plant 7:1006-1025

2. Baima S, Nobili F, Sessa G, Lucchetti S, Morelli G (1995) The expression of the Athb-8 homeobox gene is restricted to provascular cells in Arabidopsis thaliana. Development 121:4171-4182

3. Blakeslee JJ, Spatola Rossi T, Kriechbaumer V (2019) Auxin biosynthesis: spatial regulation and adaptation to stress. J Exp Bot 70:5041-5049

4. Bowman JL, Floyd SK (2008) Patterning and polarity in seed plant shoots. Annu Rev Plant Biol 59:67-88 
5. Brandt R, Salla-Martret M, Bou-Torrent J, Musielak T, Stahl M, Lanz C, Ott F, Schmid M, Greb T, Schwarz M, Choi SB, Barton MK, Reinhart BJ, Liu T, Quint M, Palauqui JC, Martínez-García JF, Wenkel $S$ (2012) Genome-wide binding-site analysis of REVOLUTA reveals a link between leaf patterning and light-mediated growth responses. Plant J 72:31-42

6. Brumos J, Robles LM, Yun J, Vu TC, Jackson S, Alonso JM, Stepanova AN (2018) Local auxin biosynthesis is a key regulator of plant development. Dev Cell 47:306-318.e305

7. Bu H, Yu W, Yuan H, Yue P, Wei Y, Wang A (2020) Endogenous auxin content contributes to larger size of apple fruit. Front Plant Sci 11:592540

8. Carlsbecker A, Lee JY, Roberts CJ, Dettmer J, Lehesranta S, Zhou J, Lindgren O, Moreno-Risueno MA, Vatén A, Thitamadee S, Campilho A, Sebastian J, Bowman JL, Helariutta Y, Benfey PN (2010) Cell signalling by microRNA165/6 directs gene dose-dependent root cell fate. Nature 465:316-321

9. Casanova-Sáez R, Voß U (2019) Auxin metabolism controls developmental decisions in land plants. Trends Plant Sci 24:741-754

10. Chen Q, Dai X, De-Paoli H, Cheng Y, Takebayashi Y, Kasahara H, Kamiya Y, Zhao Y (2014) Auxin overproduction in shoots cannot rescue auxin deficiencies in Arabidopsis roots. Plant Cell Physiol 55:1072-1079

11. Chen S, Wang X, Jia H, Li F, Ma Y, Liesche J, Liao M, Ding X, Liu C, Chen Y, Li N, Li J (2021) Persulfidation-induced structural change in SnRK2.6 establishes intramolecular interaction between phosphorylation and persulfidation. Mol Plant 14:1814-1830

12. Daudi A, O'Brien JA (2012) Detection of hydrogen peroxide by dab staining in Arabidopsis leaves. Bio-protocol 2:e263 10.21769/BioProtoc.263

13. Dello loio R, Galinha C, Fletcher AG, Grigg SP, Molnar A, Willemsen V, Scheres B, Sabatini S, Baulcombe D, Maini PK, Tsiantis M (2012) A PHABULOSA/cytokinin feedback loop controls root growth in Arabidopsis. Curr Biol 22:1699-1704

14. Donner TJ, Sherr I, Scarpella E (2009) Regulation of preprocambial cell state acquisition by auxin signaling in Arabidopsis leaves. Development 136:3235-3246

15. Du H, Liu H, Xiong L (2013) Endogenous auxin and jasmonic acid levels are differentially modulated by abiotic stresses in rice. Front Plant Sci 4:397

16. Floyd SK, Bowman JL (2006) Distinct developmental mechanisms reflect the independent origins of leaves in vascular plants. Curr Biol 16:1911-1917

17. Floyd SK, Zalewski CS, Bowman JL (2006) Evolution of class III homeodomain-leucine zipper genes in streptophytes. Genetics 173:373-388

18. Han X, Yu H, Yuan R, Yang Y, An F, Qin G (2019) Arabidopsis transcription factor TCP5 controls plant thermomorphogenesis by positively regulating PIF4 activity. iScience 15:611-622

19. Heisler MG, Ohno C, Das P, Sieber P, Reddy GV, Long JA, Meyerowitz EM (2005) Patterns of auxin transport and gene expression during primordium development revealed by live imaging of the Arabidopsis inflorescence meristem. Curr Biol 15:1899-1911 
20. Huang D, Wu W, Abrams SR, Cutler AJ (2008) The relationship of drought-related gene expression in Arabidopsis thaliana to hormonal and environmental factors. J Exp Bot 59:2991-3007

21. Ikeda Y, Men S, Fischer U, Stepanova AN, Alonso JM, Ljung K, Grebe M (2009) Local auxin biosynthesis modulates gradient-directed planar polarity in Arabidopsis. Nat Cell Biol 11:731-738

22. Kaur N, Sharma I, Kirat K, Pati PK (2016) Detection of reactive oxygen species in Oryza sativa L. (Rice). Bio-protocol 6:e2061 10.21769/BioProtoc.2061

23. Leyser $O$ (2018) Auxin signaling. Plant physiol 176:465-479

24. Li Z, Zhang X, Zhao Y, Li Y, Zhang G, Peng Z, Zhang J (2018) Enhancing auxin accumulation in maize root tips improves root growth and dwarfs plant height. Plant Biotechnol J 16:86-99

25. Liu W, Li RJ, Han TT, Cai W, Fu ZW, Lu YT (2015) Salt stress reduces root meristem size by nitric oxide-mediated modulation of auxin accumulation and signaling in Arabidopsis. Plant Physiol 168:343-356

26. Liu YG, Chen Y (2007) High-efficiency thermal asymmetric interlaced PCR for amplification of unknown flanking sequences. Biotechniques 43:649-650, 652, 654 passim

27. Mattsson J, Ckurshumova W, Berleth T (2003) Auxin signaling in Arabidopsis leaf vascular development. Plant Physiol 131:1327-1339

28. Naser V, Shani E (2016) Auxin response under osmotic stress. Plant Mol Biol 91:661-672

29. Olatunji D, Geelen D, Verstraeten I (2017) Control of endogenous auxin levels in plant root development. Int J Mol Sci 18:2587

30. Pacheco-Villalobos D, Sankar M, Ljung K, Hardtke CS (2013) Disturbed local auxin homeostasis enhances cellular anisotropy and reveals alternative wiring of auxin-ethylene crosstalk in Brachypodium distachyon seminal roots. PLoS Genet 9:e1003564 10.1371/journal.pgen.1003564

31. Paque S, Weijers D (2016) Q\&A: Auxin: the plant molecule that influences almost anything. BMC biology 14:67-67

32. Park J, Kim YS, Kim SG, Jung JH, Woo JC, Park CM (2011) Integration of auxin and salt signals by the NAC transcription factor NTM2 during seed germination in Arabidopsis. Plant Physiol 156:537549

33. Park JE, Park JY, Kim YS, Staswick PE, Jeon J, Yun J, Kim SY, Kim J, Lee YH, Park CM (2007) GH3mediated auxin homeostasis links growth regulation with stress adaptation response in Arabidopsis. J Biol Chem 282:10036-10046

34. Phillips KA, Skirpan AL, Liu X, Christensen A, Slewinski TL, Hudson C, Barazesh S, Cohen JD, Malcomber S, McSteen P (2011) vanishing tassel2 encodes a grass-specific tryptophan aminotransferase required for vegetative and reproductive development in maize. Plant Cell 23:550566

35. Ramos Báez R, Nemhauser JL (2021) Expansion and innovation in auxin signaling: where do we grow from here? Development 148:dev187120 
36. Retzer K, Weckwerth W (2021) The TOR-auxin connection upstream of root hair growth. Plants (Basel) 10:150

37. Rowe JH, Topping JF, Liu J, Lindsey K (2016) Abscisic acid regulates root growth under osmotic stress conditions via an interacting hormonal network with cytokinin, ethylene and auxin. New Phytol 211:225-239

38. Salehin M, Bagchi R, Estelle M (2015) SCFTIR1/AFB-based auxin perception: mechanism and role in plant growth and development. Plant Cell 27:9-19

39. Skirycz A, Inzé D (2010) More from less: plant growth under limited water. Curr Opin Biotechnol 21:197-203

40. Smith ZR, Long JA (2010) Control of Arabidopsis apical-basal embryo polarity by antagonistic transcription factors. Nature 464:423-426

41. Stepanova AN, Robertson-Hoyt J, Yun J, Benavente LM, Xie DY, Dolezal K, Schlereth A, Jürgens G, Alonso JM (2008) TAA1-mediated auxin biosynthesis is essential for hormone crosstalk and plant development. Cell 133:177-191

42. Stepanova AN, Yun J, Robles LM, Novak O, He W, Guo H, Ljung K, Alonso JM (2011) The Arabidopsis YUCCA1 flavin monooxygenase functions in the indole-3-pyruvic acid branch of auxin biosynthesis. Plant Cell 23:3961-3973

43. Tu M, Wang X, Yin W, Wang Y, Li Y, Zhang G, Li Z, Song J, Wang X (2020) Grapevine VlbZIP30 improves drought resistance by directly activating $V_{V N A C 17}$ and promoting lignin biosynthesis through the regulation of three peroxidase genes. Hortic Res 7:150

44. Ursache R, Miyashima S, Chen Q, Vatén A, Nakajima K, Carlsbecker A, Zhao Y, Helariutta Y, Dettmer J (2014) Tryptophan-dependent auxin biosynthesis is required for HD-ZIP III-mediated xylem patterning. Development 141:1250-1259

45. Vanstraelen M, Benková E (2012) Hormonal interactions in the regulation of plant development. Annu Rev Cell Dev Biol 28:463-487

46. Wang S, Bai Y, Shen C, Wu Y, Zhang S, Jiang D, Guilfoyle TJ, Chen M, Qi Y (2010) Auxin-related gene families in abiotic stress response in Sorghum bicolor. Funct Integr Genomics 10:533-546

47. Yamada M, Greenham K, Prigge MJ, Jensen PJ, Estelle M (2009) The TRANSPORT INHIBITOR RESPONSE2 gene is required for auxin synthesis and diverse aspects of plant development. Plant Physiol 151:168-179

48. Yang ZB, Geng X, He C, Zhang F, Wang R, Horst WJ, Ding Z (2014) TAA1-regulated local auxin biosynthesis in the root-apex transition zone mediates the aluminum-induced inhibition of root growth in Arabidopsis. Plant Cell 26:2889-2904

49. Yoshikawa T, Ito M, Sumikura T, Nakayama A, Nishimura T, Kitano H, Yamaguchi I, Koshiba T, Hibara K, Nagato Y, Itoh J (2014) The rice FISH BONE gene encodes a tryptophan aminotransferase, which affects pleiotropic auxin-related processes. Plant J 78:927-936

50. Zhang SW, Li CH, Cao J, Zhang YC, Zhang SQ, Xia YF, Sun DY, Sun Y (2009) Altered architecture and enhanced drought tolerance in rice via the down-regulation of indole-3-acetic acid by 
TLD1/OsGH3.13 activation. Plant Physiol 151:1889-1901

51. Zhao Y (2010) Auxin biosynthesis and its role in plant development. Annu Rev Plant Biol 61:49-64

52. Zhao Y (2018) Essential roles of local auxin biosynthesis in plant development and in adaptation to environmental changes. Annu Rev Plant Biol 69:417-435

53. Zhao Y, Christensen SK, Fankhauser C, Cashman JR, Cohen JD, Weigel D, Chory J (2001) A role for flavin monooxygenase-like enzymes in auxin biosynthesis. Science 291:306-309

54. Zheng Z, Guo Y, Novák O, Dai X, Zhao Y, Ljung K, Noel JP, Chory J (2013) Coordination of auxin and ethylene biosynthesis by the aminotransferase VAS1. Nat. Chem. Biol 9:244-246

\section{Figures}




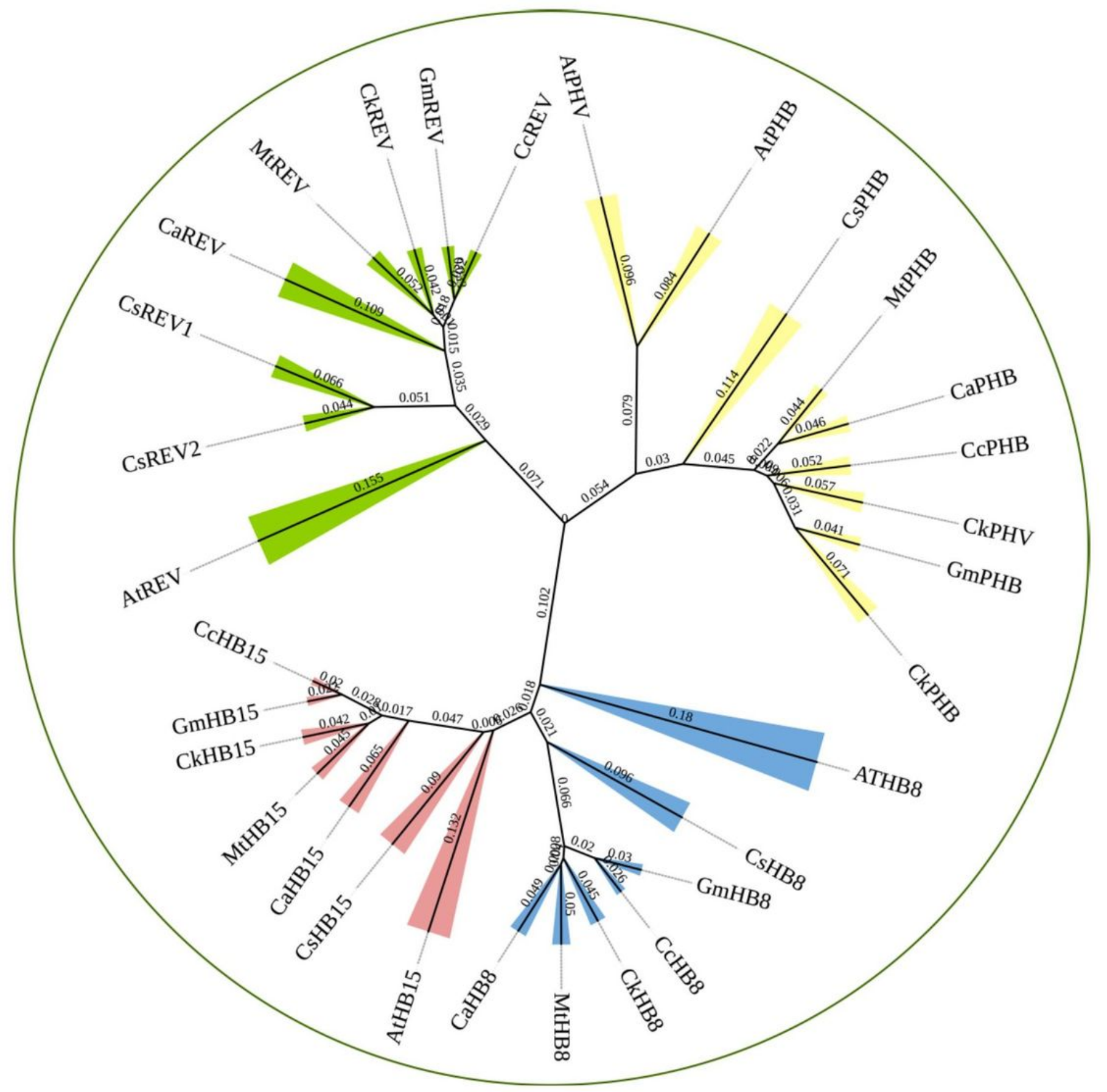

Figure 1

Phylogenetic analysis of HD-ZIP III family between C. korshinskii, G. max, C. arietinum, M. truncatula, C. cajan, $C$. sinensis and $A$. thaliana. Bootstrap support(1000 repetitions) is shown for each node 


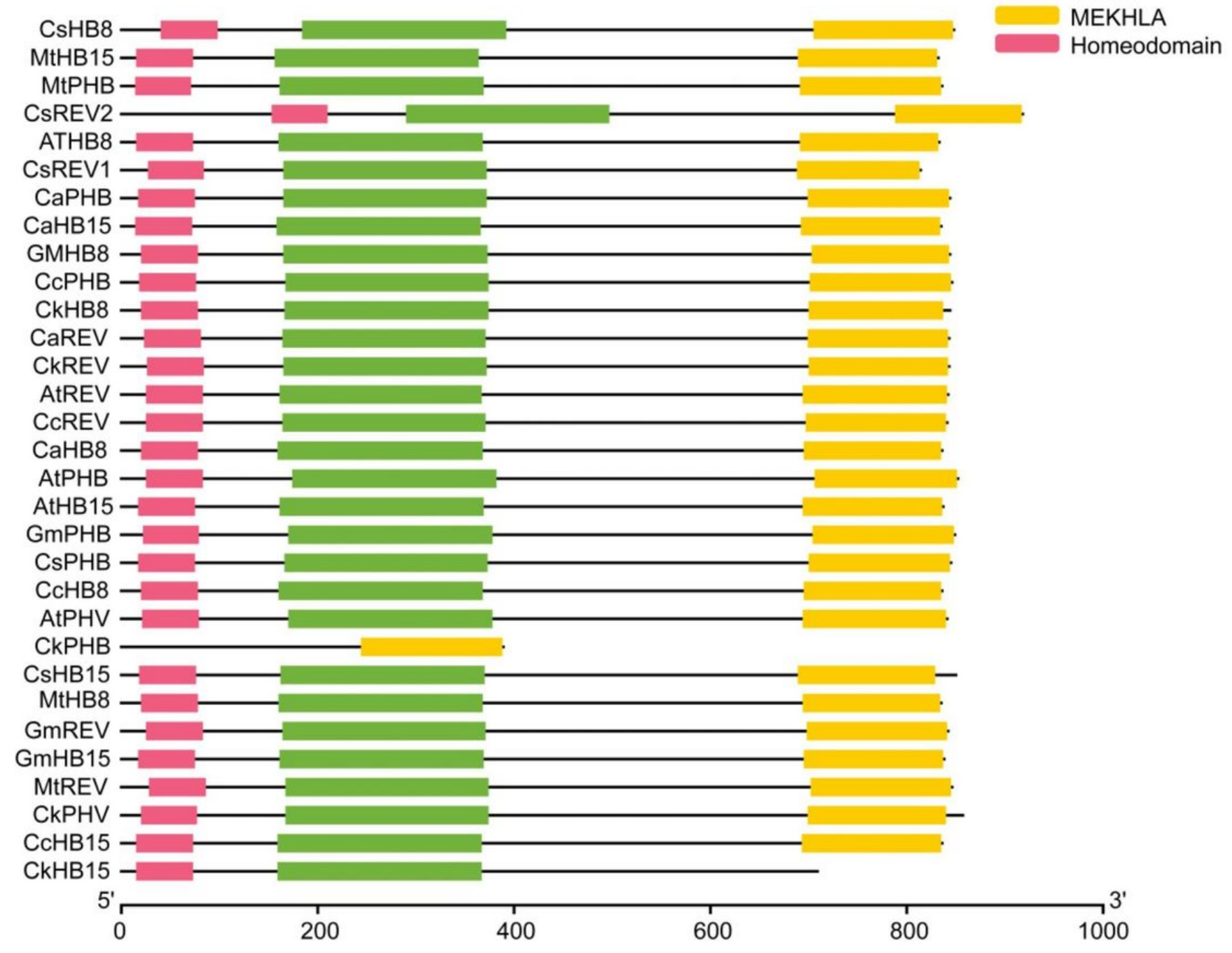

Figure 2

Conserved domain analysis of HD-ZIP III family between C. korshinskii, G. max, C. arietinum, M. truncatula, C. cajan, C. sinensis and A. thaliana 


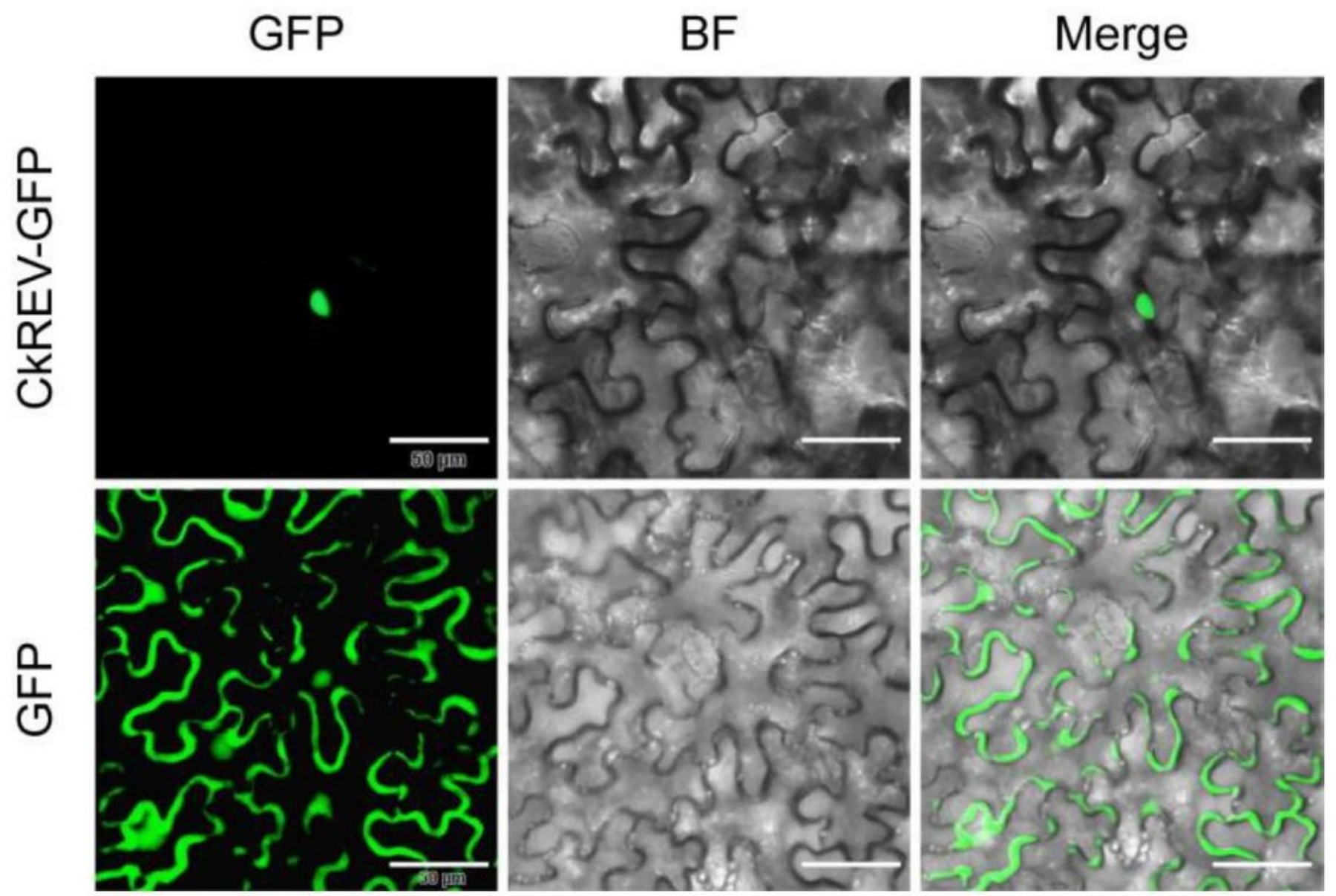

Figure 3

CkREV subcellular localization observation. Scale bars, $50 \mu \mathrm{m}$ 
(a)

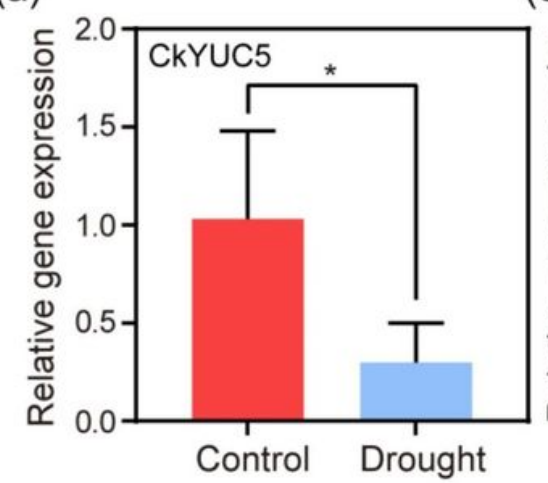

(b)

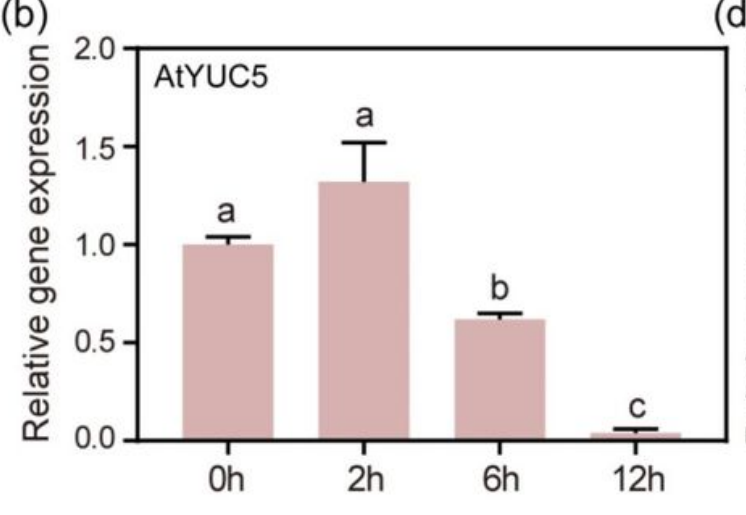

(c)

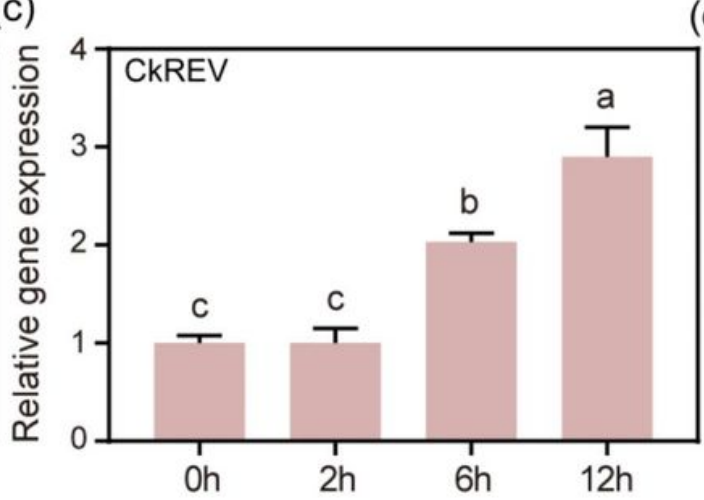

(d) (e)

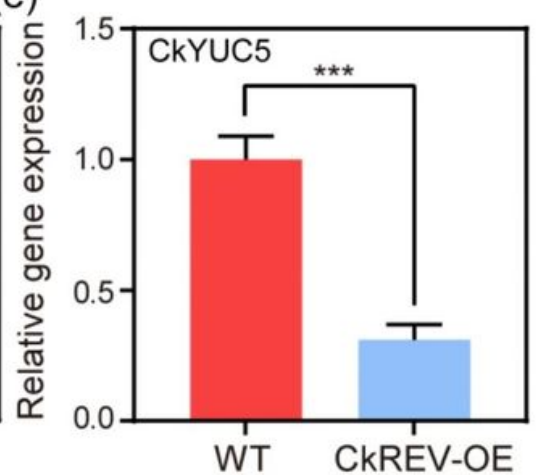

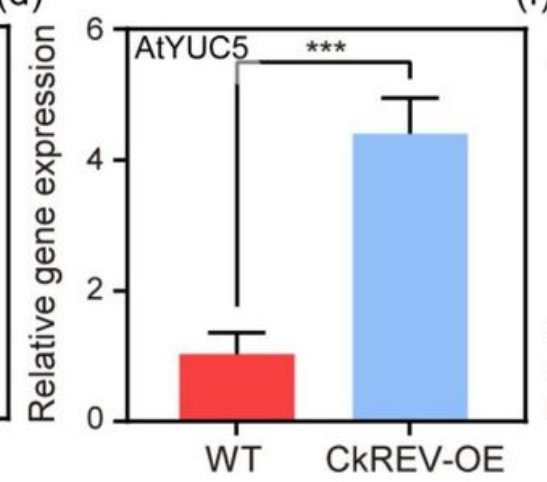

(f)

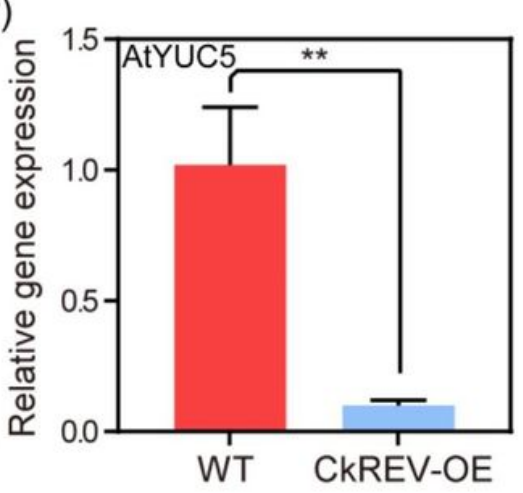

\section{Figure 4}

qRT-PCR analysis on the expression level of relevant genes. a Analysis on the expression level of $C k Y U C 5$ in C. korshinskii leaves after natural drought treatment. b Analysis on the expression level of AtYUC5 in A. thaliana seedlings after cultured on 1/2 MS medium for 7 days and transferred to PEG medium for simulated drought treatment. $\mathbf{c}$ Analysis on the expression level of $C k R E V$ in hydroponic $C$. korshinskii after drought simulation on PEG medium. $\mathbf{d}$ Analysis on the expression level of AtYUC5 in A. thaliana CKREV-OE lines at the age of 4 weeks. e Analysis on the expression level of CkYUC5 in C. korshinskii leaves instantaneously overexpressing CkREV. $\mathbf{f}$ Analysis on the expression level of AtYUC5 in wild type and transgenic $A$. thaliana after drought treatment. $\mathbf{a}, \mathbf{d}$, e and $\mathbf{f}$ Data are shown as the mean $\pm S D$ of three independent experiments. Student's t test is employed to measure statistical significance between two samples with confidence level at $0.95(*, P<0.05$; $* *, P<0.01$; $* \star *, P<0.001)$. b, $\mathbf{c}$ Data are shown as the mean $\pm S D$ of three independent experiments. One-way ANOVA was performed for the statistical analysis, where different letters represent significant differences $(P<0.05)$. Same as below 
(a)

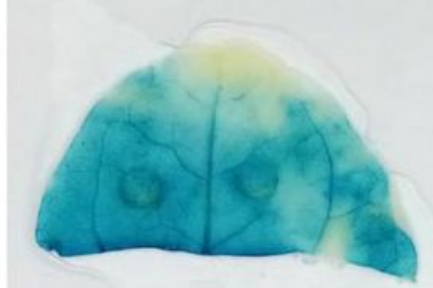

proCkYUC5:GUS + GFP (b)

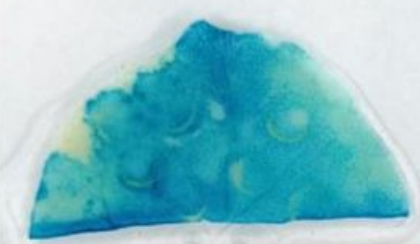

proCkYUC5:GUS $\overline{10 \mathrm{~mm}}+$ CkREV-GFP

(c) (d)

drought

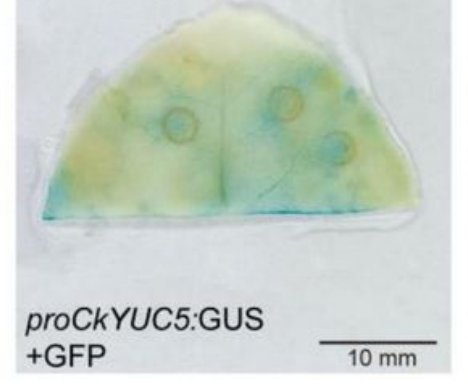

CkREV-OE
proCkYUC5:GUS

+CkREV-GFP

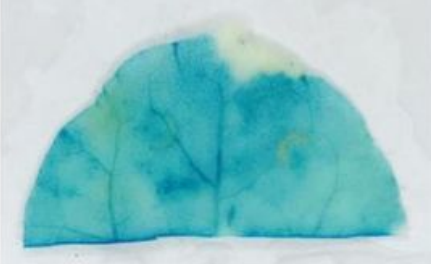

CkREV-OE

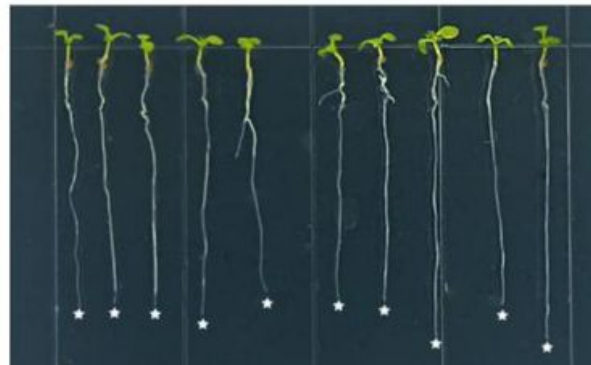

(e) Control

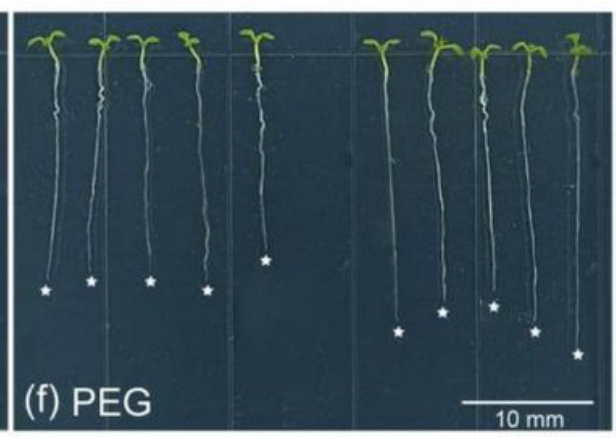

(i)

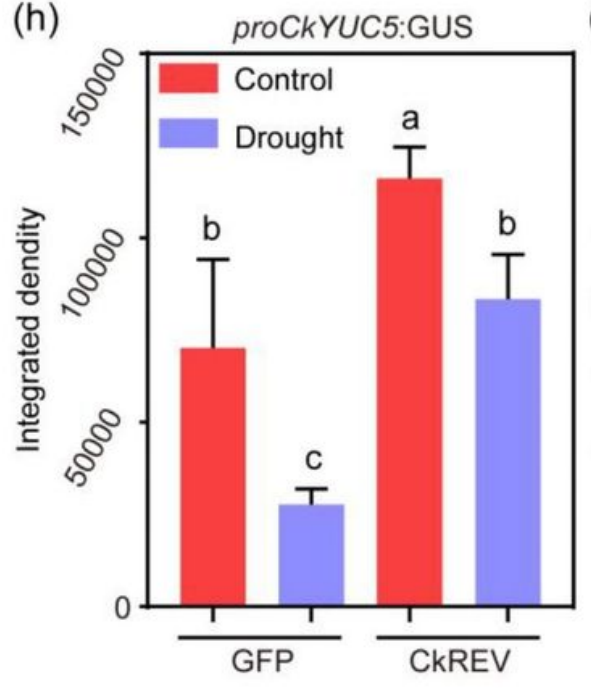

(i)

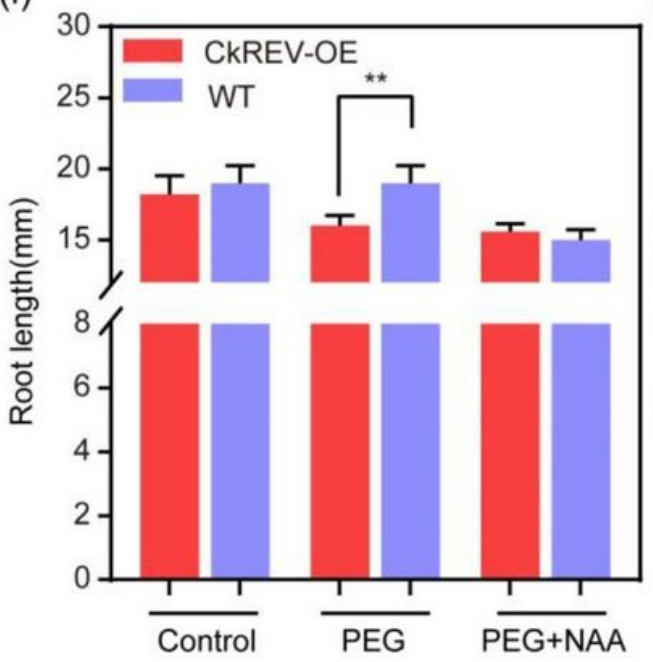

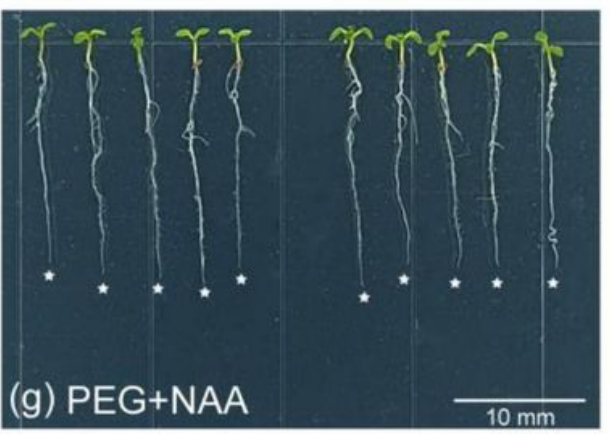

(j)

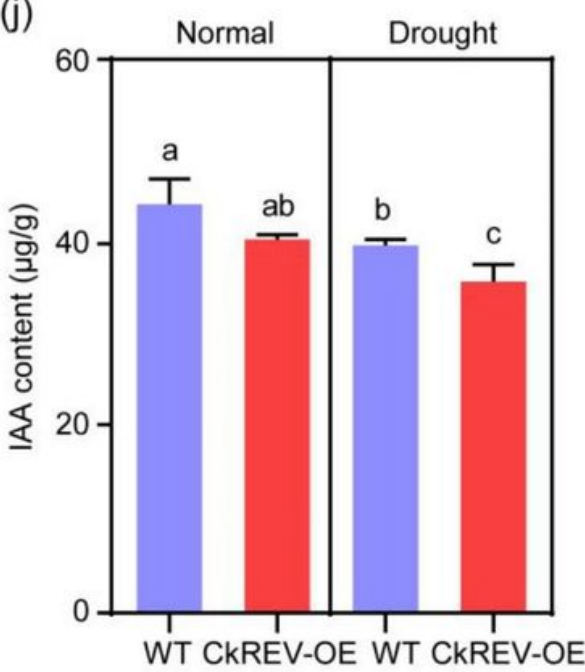

\section{Figure 5}

CkREV bidirectionally regulates the expression of CkYUC5 and inhibits the $A$. thaliana root length under drought stress. a-d Observe the expression changes of CkYUC5 under different treatment conditions through tobacco leaves cultured for 28 days ( $n=3$ biologically independent samples). a Localization of CkYUC5 in the tip of tobacco leaf under normal culture conditions. b Localization of CkYUC5 in the tip of tobacco leaf after CKREV overexpression. c Localization of CKYUC5 in the tip of tobacco leaf after PEG treatment. $\mathbf{d}$ Localization of $C k Y U C 5$ in the tip of tobacco leaf after overexpression of $C k R E V$ treated with PEG. e, $\mathbf{f}$ and $\mathbf{g}$ Four days after germination on 1/2MS plates, the phenotype of root length change of $A$. thaliana CKREV-OE strain and wild type under normal culture conditions, PEG treatment and PEG treatment with NAA added for 3 days ( $n=5$ biologically independent samples). $\mathbf{h}$ GUS staining statistics of 
A. thaliana CKREV-OE strain and wild type under different treatments. i Root length statistics of $A$. thaliana CKREV-OE strain and wild type under different treatments. $\mathrm{j}$ IAA content determination. $\mathrm{h}$ and $\mathrm{j}$ Data are shown as the mean $\pm S D$ of three independent experiments. i Data are shown as the mean $\pm S D$ of five independent experiments. Scale bars in a-g, $10 \mathrm{~mm}$

(a)

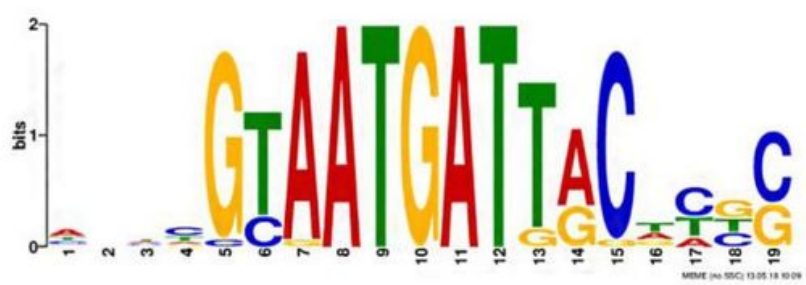

(c)

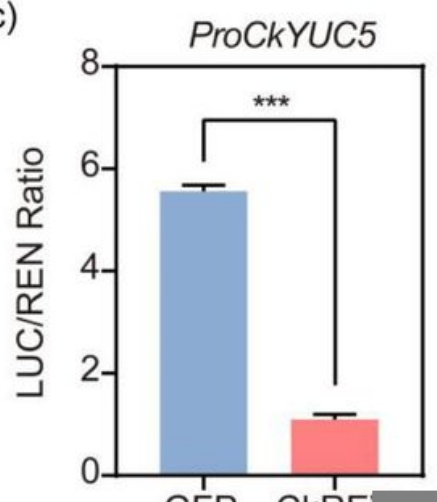

(d)

(f) (b)
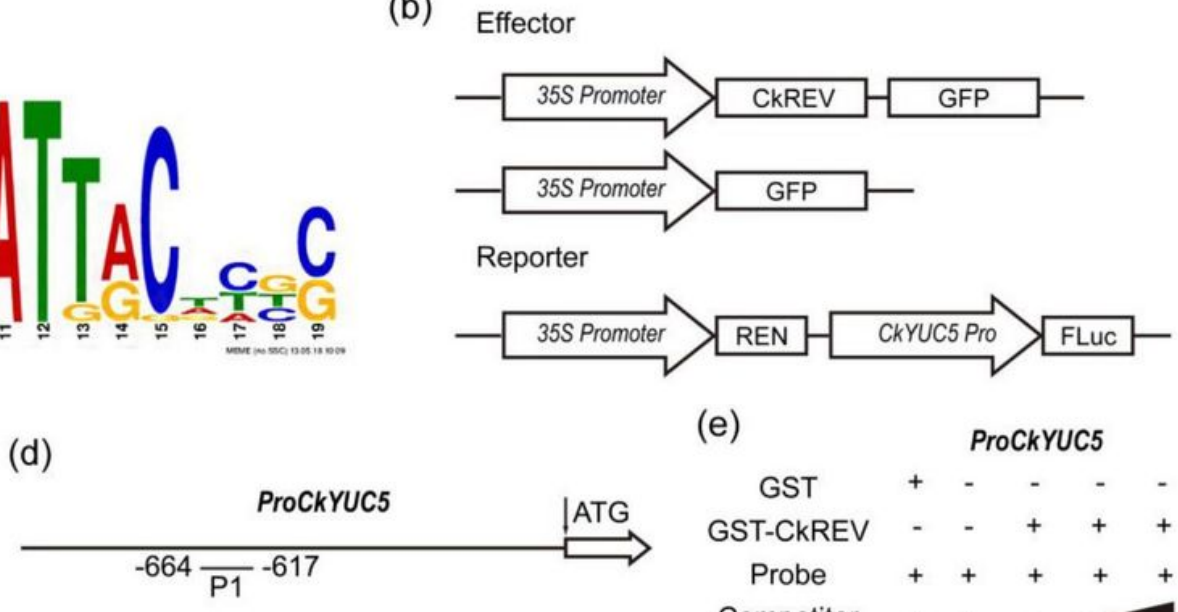

$\frac{\text { CkYUC5 }}{\text { SD-LTH(20mM) }}$

pGADT7 D 畒 (e)
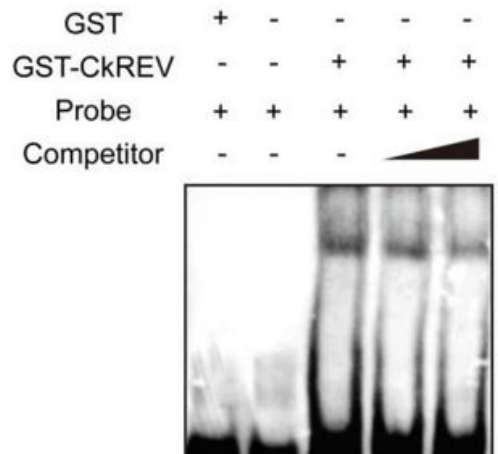

\section{Figure 6}

Interaction proof between CkREV and CkYUC5. a Recognition site of downstream genes of REV. $\mathbf{b}$ DualLUC experimental mode diagram. c Results of Dual-LUC experiment CkREV interacted with the promoter region of $C k Y U C 5$ and negatively regulated its expression compared with GFP control group. $\mathbf{d}$ Sketch map of probe binding site in EMSA experiment. e Results of EMSA experiment indicated that CkREV-GST could directly bind to ATGAT element in the promoter region of critical enzyme gene CkYUC5 in auxin synthesis. $f$ The results of yeast one-hybrid further verified the binding of CkREV to the promoter region of 
CkYUC5. g qRT-PCR detection on CkAS1 expression level in C. korshinskii leaves under different treatments. $\mathbf{h}$ Dual-LUC experimental mode diagram. $\mathbf{i}$ The Dual-LUC experiment was used to detect the effect of CkAS1 as an effector on the regulation of CkREV on CkYUC5. c, e, $g$ and $i$ Data are shown as the mean $\pm S D$ of three independent experiments.

(a)

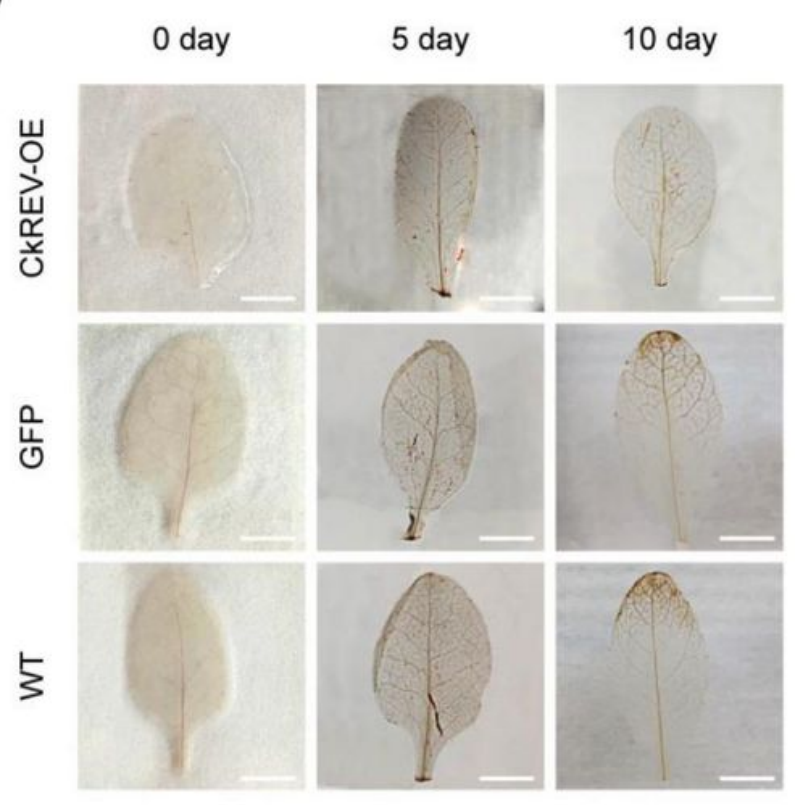

(c)

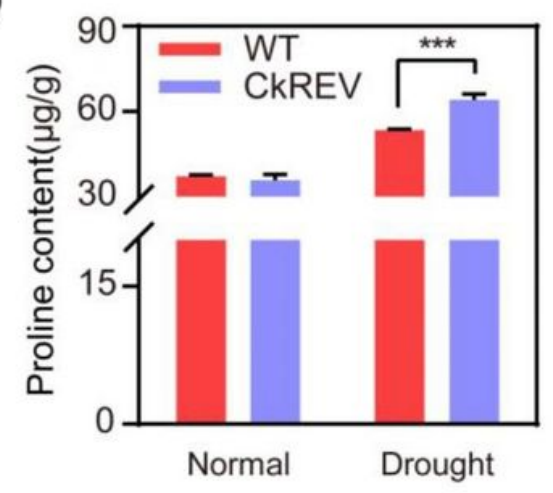

(b)

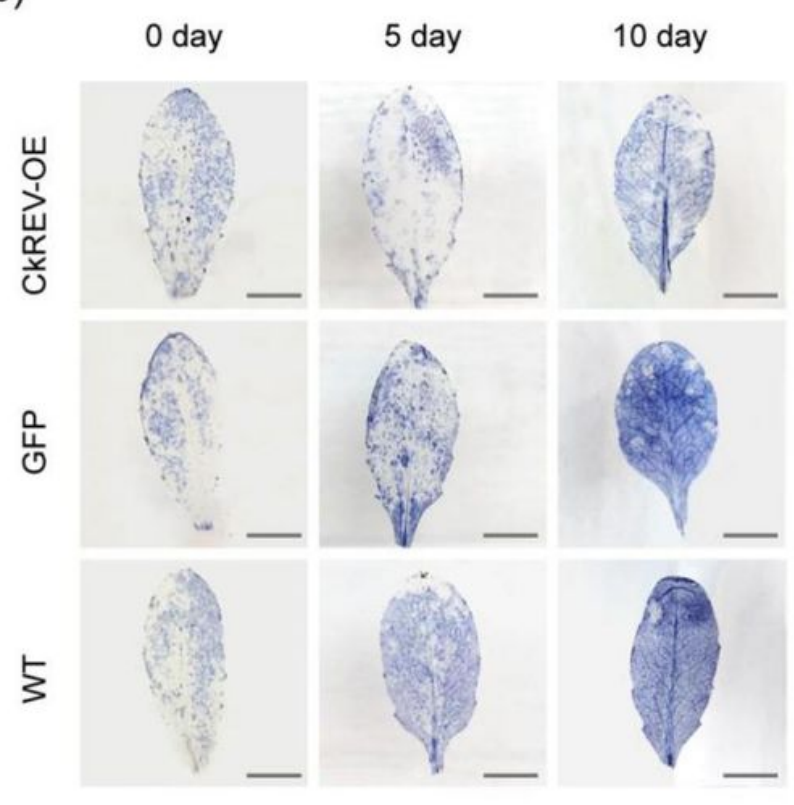

(d)

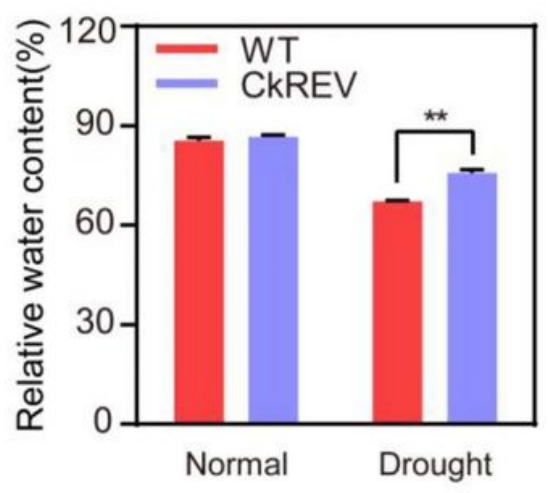

\section{Figure 7}

Phenotype analysis on transgenic $A$. thaliana. a DAB staining on rosette leaves at the same position after natural drought treatment for 0,5 and 10 days ( $n=3$ biologically independent samples). b NBT staining on rosette leaves at the same position after natural drought treatment for 0,5 and 10 days ( $n=3$ biologically independent samples). $\mathbf{c}$ Results of proline content determination. $\mathbf{d}$ Results of relative water content determination. $c$ and $d$ Data are shown as the mean $\pm S D$ of three independent experiments. Scale bars in a and b, $5 \mathrm{~mm}$ 


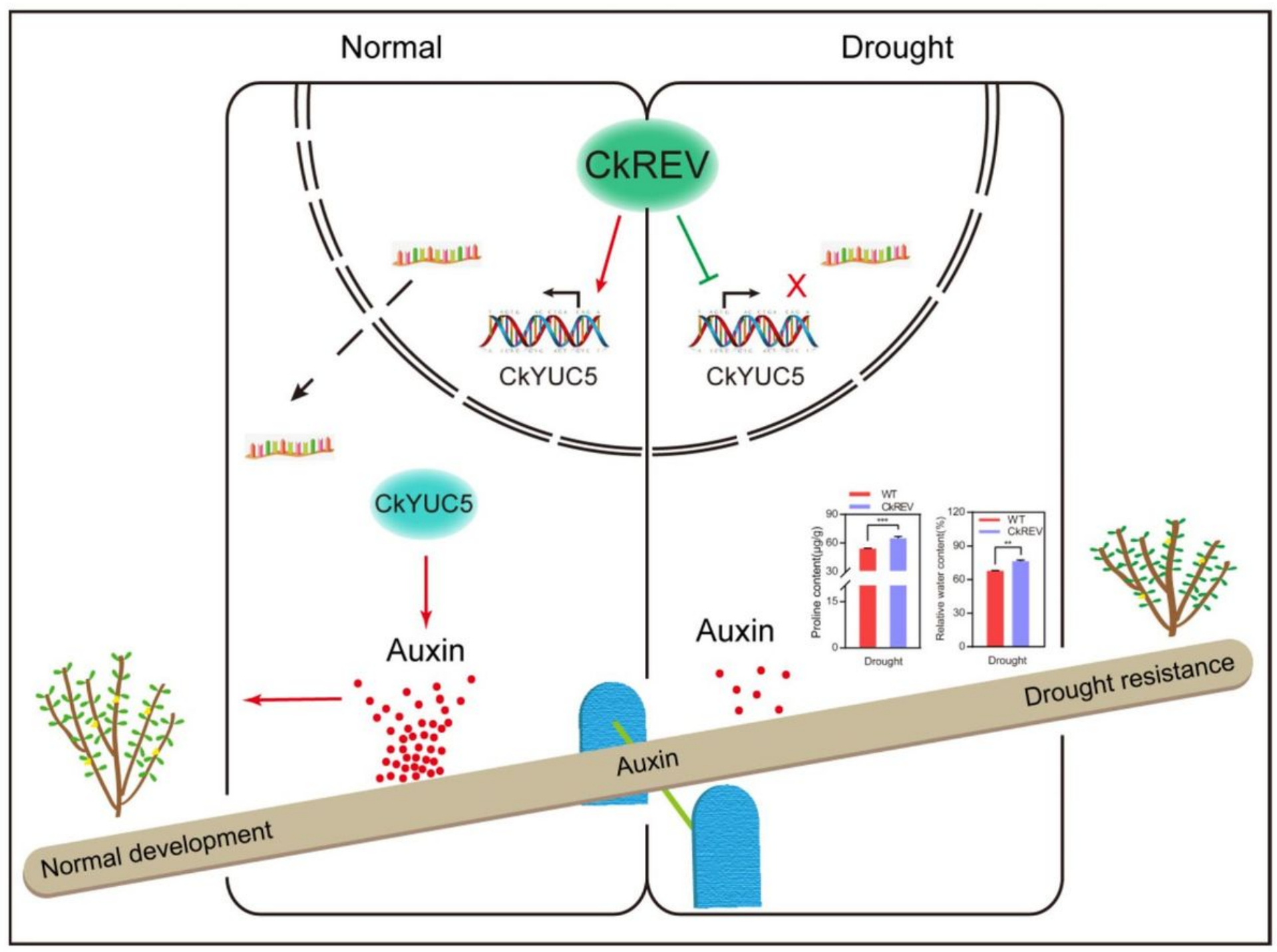

Figure 8

Model of regulation of CkREV on the expression of CkYUC5. The model concludes our research and exhibits that CkREV bidirectionally regulate the expression of CkYUC5 critical in auxin synthesis depending on changes of external environmental signals, balancing the growth and drought-resistance of plants by influencing auxin synthesis

\section{Supplementary Files}

This is a list of supplementary files associated with this preprint. Click to download.

- SupplementaryFiguresS1S3.docx

- SupplementarytablesS1S2.docx 